\title{
Simulação in Situ, uma Metodologia de Treinamento Multidisciplinar para Identificar Oportunidades de Melhoria na Segurança do Paciente em uma Unidade de Alto Risco
}

\author{
In Situ Simulation, a Multidisciplinary \\ Training Method to Identify Opportunities \\ to Improve Patient Safety Improvement in \\ a High Risk Unit
}

Regina Mayumi Utiyama Kaneko Thomaz Bittencourt Couto ${ }^{I I}$ Michael Medeiros Coelho ${ }^{I}$ Alexandre Kiyoshi Taneno ${ }^{I}$ Nádia Neves Barduzzi Joyce Kelly Silva Barreto ${ }^{I}$ Euma Ferreira de Sousal Milene Vidal da Silva Barbosa Felipe Spinelli de Carvalho ${ }^{I}$

PALAVRAS-CHAVE

- Simulação;

- Simulação de paciente;

- Equipe de Assistência ao Paciente;

- Capacitação de Recursos Humanos em Saúde;

- Educação Médica.

Introdução: A simulação é uma metodologia usada para substituir ou amplificar experiências reais por experiências guiadas que evocam ou replicam aspectos do mundo real de maneira interativa. A simulação in situ leva essa técnica diretamente aos locais onde ocorrem atendimentos, com a própria equipe de saúde atuando em seu ambiente de trabalho em cenário simulado. Objetivo: Descrever experiência piloto de simulação in situ realizada em unidade de pronto atendimento, destacando oportunidades de avaliação de sistema de atendimento, trabalho em equipe e detecção de ameaças latentes à segurança (ALS). Métodos: Estudo aplicado na Unidade Ibirapuera do Hospital Israelita Albert Einstein e realizado pelo Centro de Simulação Realística Albert Einstein. Foi apresentado cenário de paciente de 45 anos com síndrome coronariana aguda que evolui para parada cardiorrespiratória. Simulação híbrida de 30 minutos com uso de ator e simulador de alta fidelidade (SimMan 3G ${ }^{\circledR}$ ).Utilizado checklist e filmagem para avaliar habilidades e atitudes, usados em debriefing estruturado com uma hora de duração. Resultados: A experiência proporcionou avaliação técnica, comportamental e sistemas. Detectou quatro ALS e permitiu reflexão guiada sobre trabalho em equipe. Conclusão: Este piloto contribuiu para o alcance dos objetivos propostos com o cenário e demonstrou oportunidades de treinamento e melhoria. A simulação in situ pode ser usada no futuro sistematicamente para treinamento contínuo de equipes, visando à melhoria da qualidade de atendimento è̀ segurança do paciente. 


\section{KEYWORDS:}

- Simulation;

- Patient Simulation;

- Patient Care Team;

- Health Human Resource Training;

- Medical Education.

Recebido em: 13/02/2014

Aprovado em: 10/03/2015
Introduction:Simulation is a method used to substitute or enhance real experiences with guided, interactive experiences that are reminiscent of or replicate aspects of the real world.In situ simulation takes this technique directly to the places where health care is rendered, with the health team acting in a simulated scenario within their work environment.Objective:To describe the pilot in situ simulation experience conducted at an accident and emergency unit, highlighting the opportunities to assess the care system, teamwork and detection of latent safety threats (LSTs).Methods:The study was applied at the Ibirapuera Unit of the Hospital Israelita Albert Einstein and performed by the Albert Einstein Realistic Simulation Centre.A scenario was presented wherein a 45-year old patient whose acute coronary syndrome evolves into cardiac arrest. A 30-minute hybrid simulation involving the use of an actor and high fidelity simulator (SimMan 3G®).A checklist was used and the simulation was filmed in order to evaluate skills and attitudes in a structured 1-hour long debriefing.Results:The experience provided a technical, behavioural and system assessment. Four LSTs were detected and it enabled a guided reflection on teamwork. Conclusion:This pilot contributed to achieving the objectives proposed with the scenario and demonstrated opportunities for training and improvement.In situ simulation may be used systemically in the future for continuous team training, aimed at improving service quality and patient safety. 


\section{INTRODUÇÃO}

A simulação realística é uma metodologia de treinamento para substituir ou amplificar experiências reais por experiências guiadas que evocam ou replicam aspectos substanciais do mundo real de maneira interativa. Existem vários modelos de simulador, desde manequins anatômicos simples até altamente avançados, guiados por computador. A fidelidade da simulação depende do quanto esta se aproxima da realidade ${ }^{1}$.

A simulação de alta fidelidade tem sido usada na saúde como estratégia para treinamento de indivíduos e equipes em capacidades técnicas (conhecimento e habilidades) e não técnicas (comunicação, atitude e trabalho em equipe) ${ }^{1}$.

A simulação in situ leva essa técnica diretamente aos locais onde ocorre a atuação médica. Ao invés de realizar esse treinamento no centro de simulação, com equipes que nem sempre atuam juntas, a própria equipe de saúde atua em seu ambiente de trabalho em cenário simulado. Isto permite também avaliar a competência do sistema e condições latentes que podem favorecer o erro - as chamadas ameaças latentes à segurança ${ }^{2}$.

O Institute of Medicine, em seu clássico relatório To err is human ${ }^{3}$, identificou o pronto-socorro como a área de maior possibilidade de eventos adversos. Esse relato demonstrou grande risco associado à prática médica e recomendou treinamento em equipes com uso de simulação como possível ferramenta para minimizar esses eventos.

Em estudo que avalia o impacto da simulação na segurança do paciente em departamento de emergência pediátrico, Patterson et al. ${ }^{4}$ demonstraram mudança de atitude da equipe multidisciplinar em relação a eventos adversos e significativa redução desses eventos.

O presente estudo relata uma experiência inédita no Brasil: o uso de simulação in situ para treinamento de equipes e identificação de ameaças à segurança latentes em unidade de pronto atendimento.

\section{OBJETIVO}

Descrever experiência piloto de simulação in situ realizada em unidade de pronto atendimento, destacando oportunidades de avaliação do sistema de atendimento, trabalho em equipe e deteç̧ão de ameaças latentes à segurança.

\section{MÉTODOS}

\section{Local do estudo}

A simulação foi realizada na Unidade Ibirapuera do Hospital Israelita Albert Einstein. Inaugurada em 2006, conta com unidade diagnóstica e pronto atendimento. Este é equipado para o atendimento de urgência e emergência, sendo que casos que necessitem internação, cirurgia ou cuidados intensivos são es- tabilizados no local e posteriormente transferidos para o Hospital Israelita Albert Einstein, no Morumbi.

Este estudo foi aprovado pela comitê de ética e pesquisa do Hospital Israelita Albert Eisntein.

\section{Participantes}

Para não interromper o fluxo de atendimento nessa primeira experiência em simulação in situ, optou-se por chamar a equipe que não estava atuando naquele momento para conduzir a simulação. A equipe era composta de dois médicos, um enfermeiro e dois técnicos de enfermagem. Estavam de prontidão em horário de pouco fluxo habitual, sem, porém, saber detalhes sobre o caso planejado.Se a equipe de atendimento solicitasse ajuda, outros profissionais do plantão poderiam ser acionados.

\section{Instrutores}

Participaram desta atividade como instrutores um médico e uma enfermeira que não atuam nesta unidade de pronto atendimento. Eles foram convidados para acompanhar todo o fluxo, desde a chegada do ator na recepção até o término do atendimento com checklist específico para esta assistência.

Foram selecionados dois observadores, que acompanharam os instrutores para auxiliá-los no monitoramento dos tempos de cada etapa.

\section{Simulação}

Para melhor avaliar o fluxo de pacientes na unidade, desde abertura da ficha, passando pela triagem até o atendimento em si, optou-se por simulação híbrida, com uso de ator até a perda da consciência e simulador de alta fidelidade (SimMan $3 \mathrm{G} \AA$, simulador adulto da Laerdal) a partir do momento em que o ator perde a consciência e inicia o quadro de fibrilação ventricular no cenário.

Os objetivos do cenário proposto eram os seguintes:

- Avaliar riscos potenciais à segurança do paciente;

- Avaliar a aderência aos protocolos institucionais e diretrizes das práticas assistenciais baseadas em evidências;

- Analisar e prover discussão acerca das competências comportamentais no ambiente real de trabalho;

- Acompanhar e avaliar o desempenho técnico da equipe no atendimento ao paciente;

- Avaliar e sugerir melhorias nos fluxos e processos;

- Avaliar estrutura física, utilização de equipamentos e recursos humanos adequados ao atendimento dos pacientes.

Uma semana antes da simulação in situ, foi realizado um teste para alinhamento do cenário, dos instrutores, orientações 
referentesà atuação do ator e sua fala,escolha do simulador, logística de transporte de simuladores, avaliação prévia da unidade e responsabilidades de cada membro da simulação realística. $\mathrm{O}$ ator foi orientado a responder aos questionamentos dos profissionais somente se perguntado.

\section{Descrição do cenário}

\section{Fase I: ator}

Simulação iniciada na recepção da unidade.

Paciente masculino, 45 anos, na recepção, dor epigástrica leve, retirou a senha e aguardou o atendimento administrativo.
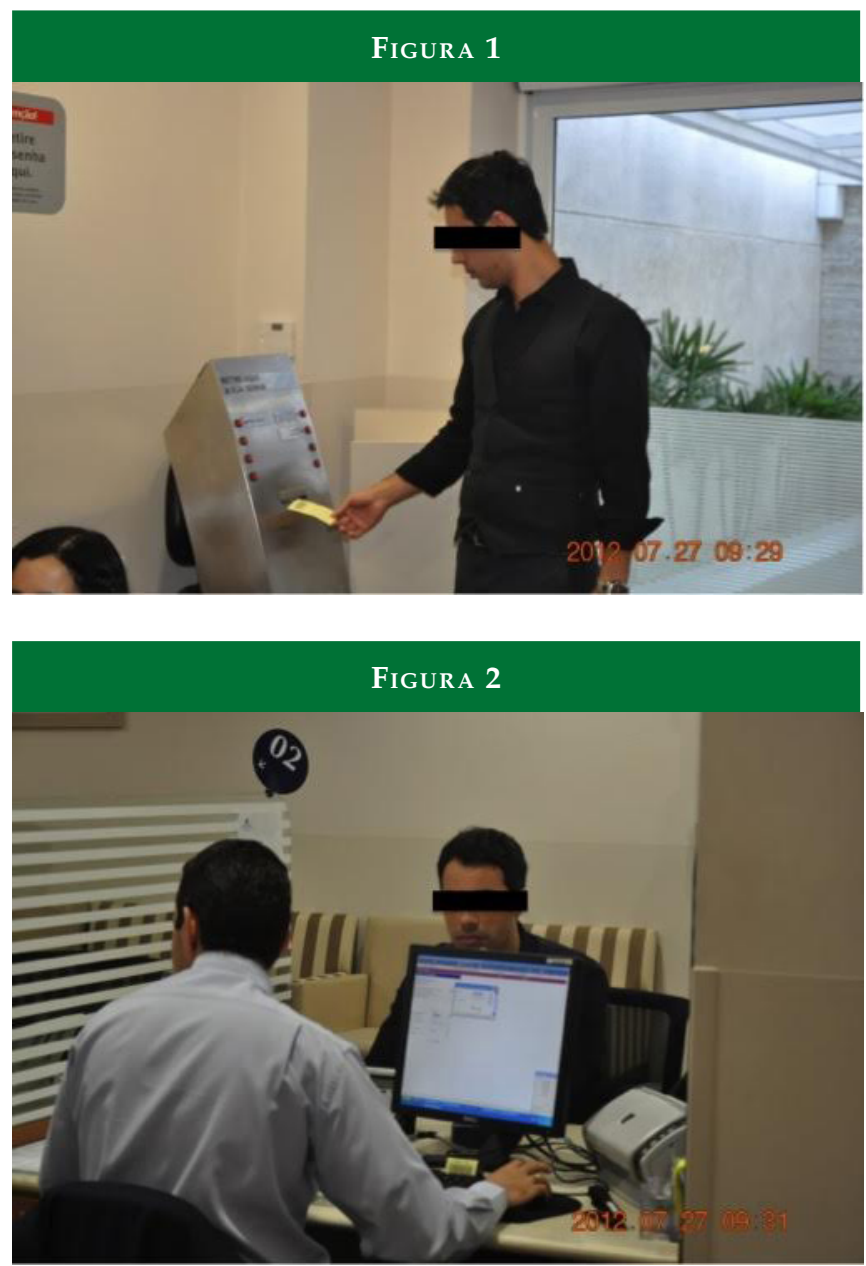

Condutas esperadas:

- Abertura do cadastro;

- Encaminhamento à triagem.

\section{Fase II: ator}

Simulação seguida com o encaminhamento do ator à sala de triagem, realizada pelo enfermeiro.
Queixa de dor epigástrica, com irradiação para o pescoço e membro superior esquerdo há uma hora, durante atividade física. No momento da admissão, referiu dor leve.

Antecedentes de tabagismo, hipertensão arterial sistêmica em uso de atenolol 50mg por dia e história familiar positiva (mãe faleceu por infarto agudo do miocárdio aos 42 anos de idade).

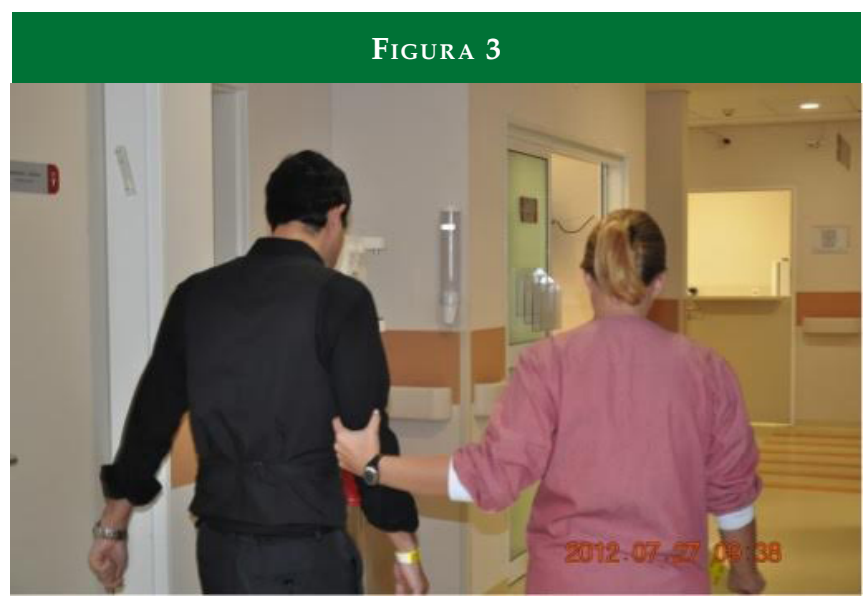

Condutas esperadas:

- Realização da triagem;

- Abertura da ficha de dor torácica;

- Encaminhamento à sala de emergência.

\section{Fase III: híbrida (ator/simulador)}

Encaminhamento do ator à sala de emergência.

Eletrocardiograma do paciente com supradesnivelamento de segmento ST em derivações V1 a V6.

Após realização de eletrocardiograma (ECG) e monitorização, o paciente evolui para parada cardiorrespiratória.

Parâmetros do simulador:

SimMan 3G® vestido com calça social e camisa, a mesma vestimenta do ator. Ao ser monitorizado, ritmo Fibrilação Ventricular (FV); demais parâmetros não detectáveis.

Condutas esperadas:

- Monitorização dos sinais vitais e realização do eletrocardiograma;

- Acionamento do médico plantonista;

- Sequência de atendimento compressões - via aérea boa ventilação;

- Choque não sincronizado de 200J sem melhora;

- Mais 2 minutos de ressuscitação cardiopulmonar (RCP) com novo choque; 


\begin{tabular}{|c|c|c|c|c|}
\hline \multicolumn{5}{|c|}{$\begin{array}{c}\text { QuADRo } 1 \\
\text { Checklist triagem }\end{array}$} \\
\hline \multicolumn{2}{|r|}{ Comportamento e/ou habilidades técnicas } & Realizado & Não realizado & Comentários \\
\hline \multirow{12}{*}{ 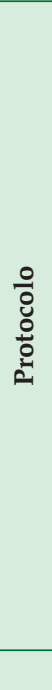 } & 1. Apresentação pessoal (nome, função e postura) & & & \\
\hline & 2. Orientações pré-triagem (objetivo) & & & \\
\hline & $\begin{array}{l}\text { 3. Realização dos SSVV (PA, FC, FR, temp.) e anotação de peso } \\
\text { e altura informados }\end{array}$ & & & \\
\hline & 4. Verificação da queixa do paciente & & & \\
\hline & $\begin{array}{l}\text { 5. Questionamento adicional (detalhar quais) sobre } \\
\text { particularidades da dor }\end{array}$ & & & \\
\hline & 6. Barreira de comunicação (linguagem) & & & \\
\hline & $\begin{array}{l}\text { 7. Questionou medicações de uso habitual (medicamento, } \\
\text { dose, horário, últimas doses) }\end{array}$ & & & \\
\hline & $\begin{array}{l}\text { 8. Histórico e antecedente - registro adequado das informações } \\
\text { da segunda página da avaliação inicial (tabagismo, } \\
\text { antecedentes familiares, uso de drogas) }\end{array}$ & & & \\
\hline & 9. Orientações de encaminhamento para sala de emergência & & & \\
\hline & 10. Relacionou queixa ao protocolo Dor Torácica. & & & \\
\hline & 11. Orientações de encaminhamento para sala de emergência & & & \\
\hline & 12. Relacionou queixa ao protocolo Dor Torácica & & & \\
\hline
\end{tabular}

- Checar sea ventilação está adequada à bolsa-valva-máscara; caso contrário, intubar;

- Se intubar - usar capnógrafo;

- Acesso venoso e adrenalina® 1 mg; (IO ou EV);

- Mais 2 minutos de RCP, trocando o socorrista;

- Novo choque (não reverte);

- Amiodarona 300 mg e mais 2 minutos de RCP, trocando o socorrista;

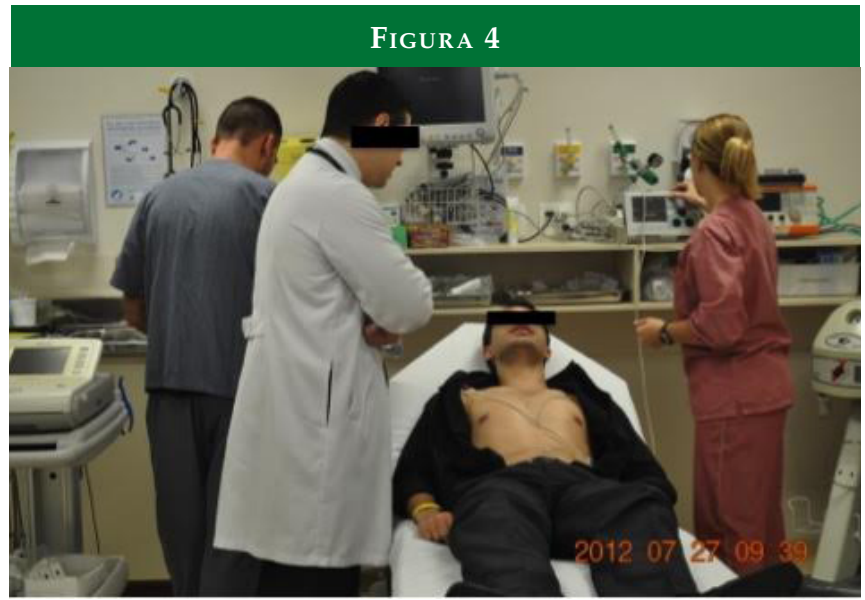

- Nova adrenalina $1 \mathrm{mg}$ e mais 2 minutos de RCP, trocando o socorrista;

- Mais um choque (não reverte);
- Mais 2 minutos de RCP e amiodarona ${ }^{\circledR} 150$ mg;

- Novo choque de 200J, com mais 2 minutos de RCP;

- Após a amiodarona® e a sequência de choques, retorno da circulação espontânea;

- Avaliação pós-parada: sinais vitais, antiarrítmico, avaliar necessidade de drogas vasoativas e transferência para unidade de tratamento intensivo.

\section{Checklists e debriefing}

O cenário foi programado para durar 30 minutos, e a discussão posterior (debriefing), com uma hora de duração, foi dividida em quatro aspectos: estrutura, materiais e equipamentos, fluxos e processos, comportamental e técnico. $\mathrm{O}$ atendimento foi filmado, o que permitiu um debriefing mais detalhado. Foram elaborados checklists estruturados para auxiliar no debriefing (Quadros 1 e 2). Participaram deste debriefing os profissionais envolvidos no atendimento, a liderança assistencial e administrativa, e os observadores.

\section{RESULTADOS}

A simulação com ator foi feita desde a chegada à unidade até a perda da consciência e início da $\mathrm{FV}$, já na sala de emergência. O tempo decorrido desde a retirada da senha até a triagem foi de 7 minutos. A triagem foi realizada em 2 minutos, e o paciente foi encaminhado à sala de emergência, andando. $\mathrm{O}$ acionamento do médico plantonista foi imediato à entrada na sala de emergência. O tempo porta ECG foi de 13 minutos. Imediata- 


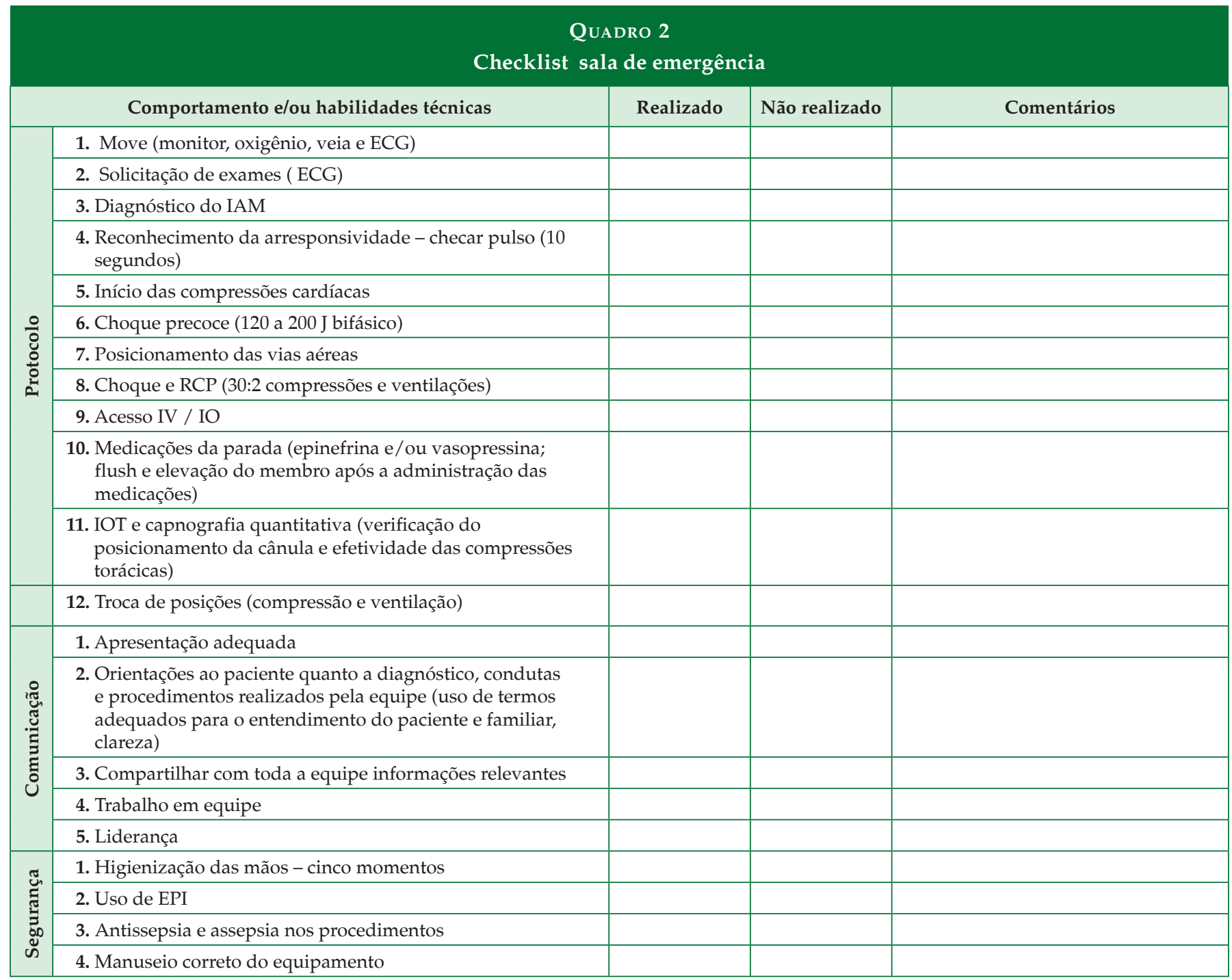

mente após o diagnóstico de infarto agudo do miocárdio, houve troca do ator pelo SimMan $3 \mathrm{G}^{\circledR}$ (este estava atrás de uma cortina que separa pacientes na sala de emergência).

Houve um momento de hesitação na condução do caso quando da troca. $\mathrm{O}$ ator já estava monitorizado e com acesso venoso puncionado. Quando passaram para o simulador, este não estava monitorizado, o que diminuiu a fidelidade do cenário.

Após esse estranhamento inicial, o manejo da parada cardiorrespiratória, de 10 minutos, ocorreu, em sua maior parte, conforme o checklist. Houve dificuldade técnica na utilização do capnógrafo (o módulo utilizado não se acoplava ao monitor), e este item foi considerado não realizado.

A compressão não foi uniforme em todos os que a realizaram, fato bem evidenciado pelo uso do vídeo e pelo feedback do simulador, demonstrando em alguns casos pouca efetividade, principalmente nos minutos finais do cenário.

Outros itens relevantes à segurança do paciente foram observados e não constavam no checklist: a prancha rígida encontrava-se no carro de emergência fora da sala de emergência, e o técnico de enfermagem necessitou sair da sala a fim de providenciar o dispositivo; e a não realização do flush e elevação do membro após a administração de adrenalina ${ }^{\circledR}$.

Após o atendimento, foi realizado debriefing estruturado, abordando-se diferentes aspectos do atendimento e usando-se como guia os checklists e a filmagem do atendimento.

\section{DISCUSSÃO}

Esta experiência com simulação in situ, até onde pudemos determinar, é pioneira no País. É uma evolução do trabalho rea- 
lizado no centro de simulação, permitindo avaliar a equipe de saúde em seu ambiente de trabalho ${ }^{5}$.

O caso apresentado proporcionou diversos aprendizados. Em termos da metodologia da simulação, definir e delimitar o tempo exato da ação no ator, ou seja, assim que encaminhado à sala de emergência, apresentar à equipe a continuidade do tratamento no simulador e descrever as competências comportamentais no checklist referentes às ações esperadas para cada competência. Seria interessante designar um observador para monitorar essas competências durante o atendimento para otimizar a discussão posterior. Além disso, foi possível avaliar o fluxo de atendimento de paciente grave desde a chegada na unidade, passando pela recepção e triagem até o atendimento da emergência.

Foram detectadas ameaças latentes à segurança:falta de treinamento da equipe da recepção na valorização de queixas dos pacientes, o que ocasionou a demora na abertura da ficha antes da triagem e excedeu o tempo porta ECG; o encaminhamento do paciente à sala de emergência andando; a localização da prancha rígida; dificuldade de ligar o capnógrafo durante a ressuscitação. A possibilidade de detectar possíveis ameaças, sejam humanas, de sistemas ou equipamentos, é um grande atrativo da simulação in situ, que foi utilizada inclusive para avaliar estruturas hospitalares e equipes de saúde antes do início das atividades com pacientes ${ }^{6,7}$. Por meio dessa atividade, foi possível discutir com as lideranças o treinamento da equipe administrativa, padronizar o encaminhamento dos pacientes à sala de emergência de cadeira de rodas e/ou maca de acordo com a classificação de risco, modificar a localização do capnógrafo, manter uma prancha rígida na sala de emergência, evitando falhas futuras e, portanto, aumentando a segurança de nossos pacientes.

A técnica detectou também um problema conhecido no atendimento da parada cardiorrespiratória: a perda da efetividade das compressões com tempo mais prolongado de atendimento ${ }^{8,9}$ e a variação da qualidade da compressão entre respondedores ${ }^{10}$. Isto possibilita treinamentos específicos futuros e reforço na técnica de ressuscitação.

A avaliação da atividade foi positiva, refletindo bom aproveitamento e possibilitando melhor integração da equipe multidisciplinar. Esta é uma potencial vantagem da simulação in situ comparada à simulação no centro de simulação: a possibilidade de treinar equipes que atuam juntas em seu ambiente de trabalho ${ }^{4,12}$.

Por ser um relato de experiência única, este estudo apresenta diversas limitações. Foi possível avaliar somente uma equipe, que não estava em suas atividades normais, mas destacada especificamente para o treinamento. $\mathrm{O}$ ideal do treina- mento com simulação in situ é ter várias oportunidades, com curta duração, em diversos horários, com a equipe que de fato atua naquele período ${ }^{11}$.

\section{CONCLUSÃO}

A simulação in situ permitiu uma oportunidade segura de avaliar e observar o desempenho da equipe multidisciplinar, tanto técnico, quanto comportamental e operacional.

A simulação in situ realizada demonstrou oportunidades de melhoria no fluxo de pacientes e atendimento de parada cardiorrespiratória. Detectou ameaças latentes à segurança - como a realização do ECG no tempo preconizado pelas diretrizes e protocolo institucional; capnógrafo inadequado ao atendimento - e permitiu revisão da técnica de ressuscitação (qualidade de compressões após minutos de RCP).

Esse método de treinamento pode ser usado no futuro sistematicamente para a capacitação contínua de equipes, visando à melhoria da qualidade de atendimento e à segurança do paciente e do ambiente.

\section{REFERÊNCIAS}

1. Gaba DM. The future vision of simulation in healthcare. Simulation in Healthcare: The Journal of the Society for Simulation in Healthcare. 2007;2(2):126-35.

2. Patterson MD, Geis GL, Falcone RA, LeMaster T, Wears RL. In situ simulation: detection of safety threats and teamwork training in a high risk emergency department. BMJ Quality \& Safety. 2012;20.

3. Committee on Quality of Health Care in America, Institute of Medicine. To Err Is Human. Kohn LT, Corrigan JM, Donaldson MS, editors. National Academies Press; 2000.

4. Patterson MD, Geis GL, LeMaster T, Wears RL. Impact of multidisciplinary simulation-based training on patient safety in a paediatric emergency department. BMJ Quality \& Safety. 2013; 21;22(5):383-93.

5. Kobayashi L, Patterson MD, Overly FL, Shapiro MJ, Williams KA, Jay GD. Educational and Research Implications of Portable Human Patient Simulation in Acute Care Medicine. Academic Emergency Medicine. 2008;15(11):1166-74.

6. Kobayashi L, Shapiro MJ, Sucov A, Woolard R, Boss RM, Dunbar J, et al. Portable advanced medical simulation for new emergency department testing and orientation. Acad Emerg Med. 2006;13(6):691-5.

7. Geis GL, Pio B, Pendergrass TL, Moyer MR, Patterson MD. Simulation to assess the safety of new healthcare teams and new facilities. Simul Healthc. 2011;6(3):125-33.

8. Berg RA, Hemphill R, Abella BS, Aufderheide TP, Cave DM, Hazinski MF, et al. Part 5: Adult Basic Life Support: 
2010 American Heart Association Guidelines for Cardiopulmonary Resuscitation and Emergency Cardiovascular Care. Circulation. 2010; 17;122(18_suppl_3):S685-S705.

9. Meaney PA, Bobrow BJ, Mancini ME, Christenson J, de Caen AR, Bhanji F, et al. CPR Quality: Improving Cardiac Resuscitation Outcomes Both Inside and Outside the Hospital: A Consensus Statement From the American Heart Association. Circulation. 2013;25.

10. Mondrup F, Brabrand M, Folkestad L, Oxlund J, Wiborg KR, Sand NP, et al. In-hospital resuscitation evaluated by in situ simulation: a prospective simulation study. Scand J Trauma Resusc Emerg Med. 2011;19:55.

11. Patterson MD, Blike GT, Nadkarni VM, Grady ML. In Situ Simulation: Challenges and Results [Internet]. Henriksen K, Battles JB, Keyes MA, Grady ML, editors. Rockville (MD): Agency for Healthcare Research and Quality (US); 2008. Available from: http://www.ncbi.nlm.nih.gov/ books/NBK43682/

12. Rosen MA, Hunt EA, Pronovost PJ, Federpwicz MA, Weaver SJ. In situ simulation in continuing educational for the health care professions. Journal of Continuing Education in the Health Professions. 2012; 32(4):243-245.

\section{CONTRIBUIÇÃO DOS AUTORES}

RMUK planejou e executou a simulação, elaborou, revisou e editou o manuscrito. TBC participou da simulação, elaborou, revisou e editou o manuscrito. MMC, AKT, NNB, JKSB, EFS, MVSB e FSC planejaram e executaram a simulação, revisaram e editaram o manuscrito.

\section{CONFLITO DE INTERESSES}

Os autores declaram não haver conflito de interesses.

\section{ENDEREÇO PARA CORRESPONDÊNCIA}

Regina Mayumi Utiyama Kaneko

Centro de Simulação Realística

Avenida Albert Einstein, 627

Morumbi - São Paulo

CEP 05652-000 - SP

E-mail: regina.utiyama@einstein.br 